\title{
Adrenal Adenoma Causing Secondary Hypertension. A Review of the Literature
}

\author{
Ali Lounici*, Esma Tabti, Abdelghani Benmekki \& Mohammed Hadi Bestaoui \\ Department of Internal Medicine, University Aboubekr Belkaid, Tlemcen, Algeria
}

Received: December 03,2018; Accepted: December 13,2018; Published: : December 17,2018

*Corresponding author : Ali Lounici, Department of Internal Medicine, University Aboubekr Belkaid, Tlemcen, Algeria. E-mail: ali.lounici@univ-tlemcen.dz

\section{Abstract}

Arterial hypertension (HTN) of endocrine origin accounts for 5 to $10 \%$ of cases of secondary hypertension. Although this form of hypertension is rare, the identification and treatment of the underlying cause could lead to healing or significant improvement in hypertension, thereby decreasing the cardiovascular risk and morbidity associated with hypertension. Endocrine causes of secondary hypertension are primary hyperaldosteronism, pheochromocytoma, Cushing's syndrome (CS), acromegaly, hyperparathyroidism, congenital adrenal hyperplasia, hypothyroidism, hyperthyroidism and secretory tumors of renin. The adrenal hypertension is the most frequent and the most frequently curable.

Epidemiological data on hypertension associated with CS are largely retrospective. However, the available data suggested that $70-$ $85 \%$ of adult patients with endogenous CS suffer from hypertension, compared to approximately $20 \%$ of patients long-term treated with exogenous corticosteroids.

The mechanisms involved in the development of hypertension during CS are complex and only partially understood. The main data concerning the renin-angiotensin system, the mineral corticoid activity, the sympathetic nervous system and the vasoregulatory system, together with the indirect mechanisms, which contribute to the development of CS-related hypertension.

The definitive therapy of CS-related hypertension is the surgical removal of the tumor responsible for the disease. Interestingly, mortality data show that adequate control of hypercortisolemia does not always lead to BP normalization. Some studies show a normalization of increased cardiovascular risk after immediate surgical cure of Cushing's disease, while others did not.

Key words: Adrenal Adenoma; Secondary Hypertension; Cushing's Syndrome

\section{Introduction}

In our observation, we describe the case of 23-year-old man with hypertension associated with paraparesis of the lower limbs revealing a Cushing syndrome due to a left adrenal adenoma. After chirurgical excision of the adrenal mass, our patient normalized his blood pressure with 3 years of follow up. This case illustrates a form of endocrine hypertension of adrenal origin and the need for a careful clinical examination during the discovery of hypertension in the young subject in order to carry out adequate balance sheets to search for a curable cause of secondary hypertension [1].

\section{Epidemiology of endocrine hypertension}

Arterial hypertension (HTN) of endocrine origin accounts for 5 to $10 \%$ of cases of secondary hypertension [2]. Although this form of hypertension is rare, the identification and treatment of the underlying cause could lead to healing or significant improvement in hypertension, thereby decreasing the cardiovascular risk and morbidity associated with hypertension. Endocrine causes of secondary hypertension are primary hyperaldosteronism, pheochromocytoma, Cushing's syndrome (CS), acromegaly, hyperparathyroidism, congenital adrenal hyperplasia, hypothyroidism, hyperthyroidism and secretory tumors of renin. The adrenal hypertension is the most frequent and the most frequently curable [3].

\section{Definition and Classification of Cushing Syndrome}

Cushing's syndrome, or chronic hypercortisolism, is a severe endocrine disease due to the prolonged exposure to glucocorticoid excess. The endogenous Cushing's syndrome, caused by the endogenous over production of cortisol by the adrenal glands, is secondary to an adrenocorticotrophin (ACTH)-secreting pituitary tumor [pituitary-dependent Cushing's syndrome (CS) or Cushing's disease (CD)] in around 70\%, a cortisol-secreting adrenal lesion (adrenal-dependent Cushing's syndrome) in 15-20\%, and an ACTH-secreting extra-pituitary tumor [ectopic Cushing's syndrome (ECS)] in 10-15\% of the cases. The chronic administration of exogenous corticosteroids for different clinical conditions may induce the development of an exogenous CS [4-6].

The diagnosis may be late and secondary to a metabolic complication. The circumstances of discovery are essentially facio-truncal obesity; signs of skin fragility, stretch marks, muscular weakness by amyotrophy, hypertension, diabetes and osteoporosis [7].

\section{Epidemiology of Hypertension Associated with CS}

Epidemiological data on hypertension associated with CS are largely retrospective. However, the available data suggested that $70-85 \%$ of adult patients $[8,9]$ with endogenous CS suffer from 
hypertension, compared to approximately $20 \%$ of patients longterm treated with exogenous corticosteroids $[10,11]$.

A particularity of hypertension associated with endogenous $\mathrm{CS}$ is the absence of a significant difference between the sexes or between the different etiologies of endogenous CS $[8,9,12]$, as well as in the degree of hypercortisolism, the blood pressure (BP) values were not found to be correlated with circulating cortisol levels $[8,13]$

HTN is present in almost $80 \%$ of patients with endogenous CS. It is most often a grade 2 hypertension (BP greater than 160 $\mathrm{mm} \mathrm{Hg}$ for systolic or $100 \mathrm{~mm} \mathrm{Hg}$ for diastolic), occurring in a young, often obese, subject with a frequent high blood pressure morning breakouts. Because of the suppression of the circadian rhythm of cortisol secretion, patients do not have nocturnal dipping [14], defined by a $10 \%$ decrease in blood pressure during sleep, which can be detected only by ambulatory blood pressure measure. Biologically, CS is defined by an increase in cortisolemia and urinary free cortisol.

\section{Mechanism of Hypertension during CS}

The mechanisms involved in the development of hypertension during CS are complex and only partially understood. The main data concerning the renin-angiotensin system (RAS), the mineralocorticoid activity, the sympathetic nervous system and the vasoregulatory system, together with the indirect mechanisms, which contribute to the development of CS-related hypertension.

The RAS is the most extensively investigated system as a putative contributor to hypertension in CS.

Confirmatory data on the involvement of the angiotensin pathways come from the acute lowering of BP obtained following oral administration of an angiotensin I-converting enzyme inhibitor (acei) in CS. The clear evidence of the angiotensin pathway involvement in the development of hypertension in CS suggests that this should be the first pharmacological target by using drugs as acei or angiotensin receptor blockers [15].

The mineralocorticoid activity has been traditionally considered a major player of hypertension secondary to glucocorticoid excess. The mineralocorticoid receptor is a nuclear receptor, mainly expressed at a renal level, which is able to bind two different corticosteroids, aldosterone and cortisol, with equal affinity [16].

In summary, renal mineralocorticoid receptor activation does not appear to be the main determinant of hypertension in most patients with CS, except in those with extremely elevated circulating cortisol levels where renal mineralocorticoid receptor hyper activation contributes to additional sodium and fluid retention, beyond the increased excretion of potassium and consequent hypokalemia. Renal mineralocorticoid receptor blockade with standard doses of spironolactone or epleronone is a reasonable adjunctive treatment in patients with hypokalemia [17].

The sympathetic nervous system, mediated by the catecholaminergic pathway, was supported only by limited controlled data. The inconsistent findings and the fact that most CS patients also develop diabetes, requiring treatment with glucose-lowering drugs, suggest that adrenergic blockade should not be a first-line treatment for CS-related hypertension.

Many substances with vasoregulatory properties have been reported to contribute to hypertension secondary to glucocorticoid excess. Endothelin-1 (ET-1), a potent vasoconstrictor. Erythropoietin (EPO) mediates glucocorticoidinduced vasoconstriction. Most studies on vasodilators have demonstrated the detrimental effects of glucocorticoid excess on the nitric oxide pathway through different mechanisms: inhibition of nitric oxide synthase (NOS) expression, reduced availability of substrates due to inhibition of the arginine transporter, or impaired cofactor generation due to inhibition of the tetrahydrobiopterin synthesis $[18,19]$. Atrial natriuretic peptide (ANP, a hormone with natriuretic vasorelaxant and RASinhibiting properties, is often reported as increased in CS [20]. CS might also be associated with impaired production of other powerful vasodilators including prostaglandins, prostacyclins and compounds of the kallikrein-kinin system, the latter due to accelerated renal kininase activity [21].

Although the prevalence of hypertension is similar among various forms of endogenous CS, however, clinical and physiopathological differences characterize CS subtypes. A tendency towards higher BP is seen in adrenal compared to pituitary tumors [17]. This difference may be related to the specific vasoactive effects of adrenal sex steroids [androgens, estrogens and their metabolites], which are generally suppressed in adrenal tumors secreting cortisol compared to pituitary tumors responsible for Cushing's disease [22, 23].

\section{Mechanism of paraparesis during CS}

The probable mechanism of the paraparesis of the lower limbs seen in our patient may be due to muscular catabolism. Cortisol causes an increase in the catabolic activity of muscle proteins [Hyper-catabolism of protein syndrome], causing muscular atrophy; it provokes paraparesis that is sometimes very debilitating. This paraparesis is often bilateral and symmetrical predominating in the thighs and can also affect the upper limbs.

Another mechanism of the paraparesis is hypokalemia, a complication that can be seen in the CS. The clinical translation varies according to the intensity of hypokalemia, it ranges from asthenia with muscle weakness to paraparesis. Patients with ectopic secretion of ACTH develop more frequently severe hypokalemia, mainly due to saturation of the activity of the 11beta-hydroxysteroid dehydrogenase 2 . The rapidity and severity of onset of hypercortisolism in this condition is responsible for severe organ damage. In extreme hypokalemia, a cases of rhabdomyolysis have been described. [24].

Spinal epidural lipomatosis which is a state of pathological fat tissue overgrowth in the vertebral canal, is an unusual cause of paraparesis in a patient on chronic steroid therapy. Cases have been reported in patients with morbid obesity, Cushing syndrome, and hypothyroidism [25]. 


\section{Effect of Treatment of CS on Hypertension}

The definitive therapy of CS-related hypertension is the surgical removal of the tumor responsible for the disease. Interestingly, mortality data show that adequate control of hypercortisolemia does not always lead to BP normalization. Some studies show a normalization of increased cardiovascular risk after immediate surgical cure of Cushing's disease [26], while others did not [27].

\section{References}

1. E Tabti, A Benmekki, MH Bestaoui, M Messad, MA Ghembaza, A Lounici. Hypertension artérielle associée à une paraparésie des membres inférieurs révélant un adénome surrénalien. Annales de Cardiologie et d'Angéiologie. 2018;67(3):226-229. Doi: 10.1016 / j.ancard.2018.05.001

2. A Velasco and $W$ Vongpatanasin, The evaluation and treatment of endocrine forms of hypertension, Curr Cardiol Rep. 2014;16(9):528610. Doi: $10.1007 / \mathrm{s} 11886-014-0528-\mathrm{x}$

3. L Amar, A Lorthioir, A Giavarini, PF Plouin and M Azizi. Presentations and management of adrenal hypertension, Presse Med 2014;43(4 pt 1):420-427. Doi: 10.1016/j.lpm.2013.06.032

4. R Pivonello, MC De Martino, LM De, G Lombardi and A. Colao, Cushing's syndrome, Endocrinol Metab Clin North Am 2008;37(1):135-149. Doi: $10.1016 /$ j.ecl.2007.10.010

5. D Graversen, P Vestergaard, K Stochholm, CH Gravholt and JO Jorgensen. Mortality in Cushing's syndrome: a systematic review and meta-analysis, Eur J Intern Med. 2012;23(2):278-282. Doi: 10.1016/j. ejim.2011.10.013

6. JK Lambert, L Goldberg, S Fayngold, J Kostadinov, KD Post and EB Geer. Predictors of mortality and long-term outcomes in treated Cushing's disease: a study of 346 patients, J Clin Endocrinol Metab. 2013;98(3):1022-1030. Doi: 10.1210/jc.2012-2893

7. G Arnaldi, A Angeli, AB Atkinson, X Bertagna, F Cavagnini, GP Chrousos, et al., Diagnosis and complications of Cushing's syndrome: a consensus statement, J Clin Endocrinol Metab. 2003;88(12):5593-5602. Doi: 10.1210/jc.2003-030871

8. T Mancini, B Kola, F Mantero, M Boscaro and G Arnaldi. High cardiovascular risk in patients with Cushing's syndrome according to 1999/WHO/ISH guidelines, Clin Endocrinol (Oxf). 2004;61(6):768777.

9. E Valassi, A Santos, M Yaneva, M Tóth, CJ Strasburger, P Chanson, et al., The European Registry on Cushing's syndrome: 2-year experience. Baseline demographic and clinical characteristics, Eur J Endocrinol. 2011;165(3):383-392. Doi: 10.1530/EJE-11-0272

10.GP Rossi, TM Seccia, C Maniero and AC Pessina. Drug-related hypertension and resistance to antihypertensive treatment: a call for action, J Hypertens. 2011;29(12):2295-2309. Doi: 10.1097/ HJH.0b013e32834c465d

11.F Hammer and PM Stewart. Cortisol metabolism in hypertension, Best Pract Res Clin Endocrinol Metab. 2006;20(3): 337-353. Doi: 10.1016/j.beem.2006.07.001

12. GF Pecori, M Moro and F Cavagnini. Gender-related differences in the presentation and course of Cushing's disease, J Clin Endocrinol Metab 2003;88(4):1554-1558. Doi: 10.1210/jc.2002-021518
13. R Giordano, A Picu, E Marinazzo, V D’Angelo, R Berardelli, I Karamouzis, et al., Metabolic and cardiovascular outcomes in patients with Cushing's syndrome of different aetiologies during active disease and 1 year after remission, Clin Endocrinol (Oxf). 2011;75(3):354-360. Doi: 10.1111/j.1365-2265.2011.04055.x

14. MA Magiakou, P Smyrnaki and GP Chrousos. Hypertension in Cushing's syndrome, Best Pract Res Clin Endocrinol Metab 2006;20(3):467-482. Doi: 10.1016/j.beem.2006.07.006

15. G Yasuda, H Shionoiri, S Umemura, I Takasaki, M Ishii. Exaggerated blood pressure response to angiotensin II in patients with Cushing's syndrome due to adrenocortical adenoma. Eur J Endocrinol. 1994;131(6):582-588.

16. PJ Fuller, MI Young. Mechanisms of mineralocorticoid action. Hypertension. 2005;46(6):1227-1235. Doi:

10.1161/01.HYP.0000193502.77417.17

17. AM Isidori, C Graziadio, RM Paragliola, A Cozzolino, AG Ambrogio, A Colao, et al. The hypertension of Cushing's syndrome: controversies in the pathophysiology and focus on cardiovascular complications. Journal of Hypertension. 2015;33(1):44-60. Doi: 10.1097/ HJH.0000000000000415

18. T. Wallerath, Witte K, Scha"fer SC, Schwarz PM, Prellwitz W, Wohlfart $P$, et al. Down-regulation of the expression of endothelial NO synthase is likely to contribute to glucocorticoid-mediated hypertension. Proc Natl Acad Sci U S A 1999;96(23):13357-13362.

19. Simmons WW, Ungureanu-Longrois D, Smith GK, Smith TW, Kelly RA. Glucocorticoids regulate inducible nitric oxide synthase by inhibiting tetrahydrobiopterin synthesis and L-arginine transport. J Biol Chem. 1996;271(39):23928-23937.

20. Knoepfelmacher M, Pradal MJ, Dio RD, Salgado LR, Semer M, Wajchenberg BL, Liberman B. Effect of corticotrophin-releasing hormone on arginine vasopressin and atrial natriuretic factor in patients with Cushing's disease. Clin Endocrinol (Oxf) 1998;49(1):7784.

21. Axelrod L. Inhibition of prostacyclin production mediates permissive effect of glucocorticoids on vascular tone. Perturbations of this mechanism contribute to pathogenesis of Cushing's syndrome and Addison's disease. Lancet 1983;1(8330):904-906.

22.JR Carter, Q Fu, CT Minson and MJ Joyner, Ovarian cycle and sympathoexcitation in premenopausal women, Hypertension 2013;61(2):395-399. Doi: 10.1161/HYPERTENSIONAHA

23. M Coylewright, JF Reckelhoff and P Ouyang. Menopause and hypertension: an age-old debate, Hypertension. 2008;5(4)1:952-959. Doi: 10.1161/HYPERTENSIONAHA.107.105742

24.E Andrawus, I Hochberg and ZS Azzam. A young patient with leg weakness and hypokalemia-case report. Rambam Maimonides Med J. 2018;9(2):e0015. Doi: 10.5041/RMMJ.10330

25. Fogel GR, Cunningham PY III, Esses SI.Spinal epidural lipomatosis: case reports, literature review and metaanalysis. Spine J. 2005;5(2):202211.

26. Hammer GD, Tyrrell JB, Lamborn KR, Applebury CB, Hannegan ET, Bell S, et al. Transsphenoidal microsurgery for Cushing's disease: initial outcome and long-term results. J Clin Endocrinol Metab 2004;89(12):6348-6357. Doi: 10.1210/jc.2003-032180

27. Clayton RN, Raskauskiene D, Reulen RC, Jones PW. Mortality and morbidity in Cushing's disease over 50 years in Stoke-on-Trent, UK: audit and meta-analysis of literature. J Clin Endocrinol Metab. 2011;96(6):632-642. Doi: 10.1210/jc.2010-1942 